\title{
Real-World Management of Hyperkalemia in the Emergency Department: An Electronic Medical Record Analysis
}

\author{
Jill Davis · Rubeen Israni - Keith A. Betts · Fan Mu (D) • \\ Erin E. Cook · Deborah Anzalone · Harold Szerlip · Lei Yin • \\ Gabriel I. Uwaifo • Eric Q. Wu
}

Received: October 29, 2021 / Accepted: December 6, 2021 / Published online: December 27, 2021

(C) The Author(s) 2021

\section{ABSTRACT}

Introduction: Hyperkalemia is often managed in the emergency department (ED) and it is important to understand how ED management and post-discharge outcomes vary by hyperkalemia severity. This study was conducted to characterize ED management and post-discharge outcomes across hyperkalemia severities. Methods: Adults with an ED visit with hyperkalemia (at least one serum potassium lab measure above $5.0 \mathrm{mEq} / \mathrm{L}$ ) were selected from US electronic medical record data (2012-2018). Patient characteristics, potassium levels, treatments, and monitoring prior to and during the ED visit were compared by hyperkalemia severity (mild [ $>5.0-5.5 \mathrm{mEq} / \mathrm{L}]$, moderate [ $>5.5-6.0]$,

Supplementary Information The online version contains supplementary material available at https:// doi.org/10.1007/s12325-021-02017-w.

J. Davis · R. Israni · D. Anzalone

Formerly AstraZeneca, Wilmington, DE, USA

K. A. Betts · F. Mu (ه) · E. E. Cook · L. Yin ·

E. Q. $\mathrm{Wu}$

Analysis Group, Inc., 111 Huntington Avenue, 14th

Floor, Boston, MA 02199, USA

e-mail: Fan.Mu@analysisgroup.com

H. Szerlip

Baylor University Medical Center, Dallas, TX, USA

G. I. Uwaifo

Ochsner Medical Center, New Orleans, LA, USA severe $[>6.0]$ ) using unadjusted analyses. Death, immediate inpatient admission, 30-day hyperkalemia recurrence, and 30-day inpatient admission were also assessed by severity.

Results: Of 6222 patients included, 4432 (71.2\%) had mild hyperkalemia, 1085 (17.4\%) had moderate, and 705 (11.3\%) had severe hyperkalemia. Chronic kidney disease (39.9-50.1\%) and heart failure (21.6-24.3\%) were common. In the $\mathrm{ED}$, electrocardiograms (mild, 56.5\%; moderate, 69.6\%; severe, 81.0\%) and patients with at least two potassium laboratory values increased with severity $(15.0 \%$; $40.4 \%$; 75.5\%). Among patients with at least two potassium laboratory values, over half of patients (60.4\%) had potassium levels $\leq 5.0 \mathrm{mEq} / \mathrm{L}$ prior to discharge. Use of potassium-binding treatments (sodium polystyrene sulfonate: mild $=4.1 \%$; moderate $=17.1 \% ; \quad$ severe $=27.4 \%), \quad$ temporizing agents $(5.6 \% ; 15.5 \% ; 31.6 \%)$, or dialysis $(0.4 \%$; $0.8 \% ; 3.0 \%)$ increased with severity; treatment at discharge was not common. Death $(1.1 \%$; $3.7 \% ; 10.6 \%)$, immediate admission to inpatient care $(5.8 \% ; 8.7 \% ; 12.7 \%)$, 30-day hyperkalemia recurrence $(2.9 \% ; 19.0 \% ; 32.5 \%)$, 30-day inpatient admission with hyperkalemia $(6.5 \% ; 7.9 \% ; 9.3 \%)$ also increased with severity. Conclusion: Patients with moderate and severe hyperkalemia experienced elevated risk of hyperkalemia recurrence and hyperkalemia-related inpatient readmission following discharge from the ED from a descriptive analysis. Future 
research to assess strategies to reduce hyperkalemia recurrence and inpatient admission in this patient population would be beneficial.

Keywords: Electronic medical records; Emergency department; Hyperkalemia; Postdischarge; Real-world outcomes

\section{Key Summary Points}

Why carry out this study?

Hyperkalemia is a potentially lifethreatening electrolyte abnormality that is associated with a substantial clinical and economic burden

Although the clinical management of hyperkalemia may necessitate treatment in the emergency department, evidence regarding patient outcomes in this setting is limited

In this study, we described demographics and clinical characteristics of patients with mild, moderate, and severe hyperkalemia who were managed in the emergency department; additionally, we describe the rates of monitoring, treatment patterns, and rates of subsequent recurrence and inpatient admissions following discharge from the emergency department

\section{What was learned from this study?}

In this descriptive analysis, patients with moderate and severe hyperkalemia experienced an increased risk of death and direct inpatient admission after an emergency department visit

Patients with moderate and severe hyperkalemia also had higher rates of hyperkalemia recurrence and hyperkalemia-related inpatient readmissions following discharge from the emergency department

Additional research to identify strategies aimed at reducing recurrence and inpatient admissions is needed

\section{INTRODUCTION}

Hyperkalemia is an electrolyte disorder defined as abnormally high levels of serum potassium [1-4]. In 2014, 1.55\% (3.7 million) of adults in the general population in the United States (US) were estimated to have hyperkalemia, with a higher prevalence $(6.35 \%)$ estimated for adults with chronic kidney disease (CKD) and/or heart failure [5]. The prevalence of hyperkalemia is expected to increase owing to the increase in prevalence of risk factors associated with hyperkalemia (e.g., heart failure, hypertension, CKD) [6]. Hyperkalemia presents a substantial economic burden, as patients with hyperkalemic events incurred \$15,606 higher 1-year total healthcare costs than patients without hyperkalemia [7].

Although mild hyperkalemia (> 5.0-5.5 mEq/L) is often asymptomatic, severe forms of hyperkalemia $(>6.0 \mathrm{mEq} / \mathrm{L})$ may be life-threatening, resulting in cardiac arrhythmias and sudden death $[2,4,8]$. However, there is large inter-individual variation and symptoms do not always correlate with the absolute potassium level observed [9]. As a result of these potentially fatal symptoms associated with very high potassium levels, patients are often managed in the emergency department (ED) where they are recommended to receive prompt and aggressive care [10, 11]. Following laboratory and electrocardiogram (ECG) assessments to confirm elevated potassium levels, treatment typically begins with agents to stabilize the membrane (e.g., calcium gluconate, calcium chloride) [9]. If there is no resolution, agents that redistribute potassium into cells (e.g., insulin and glucose or albuterol) are utilized [9], and sodium bicarbonate may be administered if metabolic acidemia occurs [9]. In the event that elevated potassium levels persist, excess levels are eliminated through use of potassium-binding agents, dialysis, and loop diuretics $[8-10,12,13]$. Ongoing monitoring of cardiac function using ECGs and laboratory assessments of serum potassium levels are also recommended for patients with moderate to severe hyperkalemia within the ED [11]. 
Evidence regarding the management of patients with hyperkalemia in the ED and outcomes following ED visits, such as hyperkalemia recurrence, is limited [14-17]. One retrospective study [16] showed that ED treatment patterns varied widely and that higher potassium levels were associated with increased rates of hospitalization and mortality. Other studies have focused on the management of patients with more severe hyperkalemia $(>6.0 \mathrm{mEq} / \mathrm{L})$, but few studies have characterized mild or moderate hyperkalemia.

Real-world evidence can help clinicians manage patients with hyperkalemia across different severities in the ED. In this study, we characterized the demographics and clinical characteristics of patients with hyperkalemia in the ED setting. We also examined the rates of monitoring and treatments administered in the ED setting as well as the rates of subsequent inpatient admission and hyperkalemia recurrence after discharge from the ED. All analyses were stratified by the severity of hyperkalemia.

\section{METHODS}

\section{Data Source}

This study was a retrospective cohort study using electronic medical record (EMR) data from the US Research Action for Health Network (REACHnet) from 2012 to 2018. REACHnet, formed in 2014, is one of nine clinical data research networks participating in PCORnet, the National Patient-Centered Outcomes Research Network, and contains EMR data for over five million patients of all ages, sexes, and racial/ethnic groups from five different health systems.

\section{Compliance with Ethics Guidelines}

The New England Independent Review Board provided written approval for this study on June 25, 2018 (NEIRB\# 1-5667-1). Written and verbal consent from participants are not applicable to this study.

\section{Sample Selection}

The study population included adult patients with at least one ED visit with hyperkalemia (at least one potassium laboratory value $>5.0 \mathrm{mEq} / \mathrm{L}$ ). Potassium laboratory results were identified on the basis of Logical Observation Identifier Names and Code (LOINC) codes and laboratory names (Supplementary Table 1). The first potassium laboratory value during the $\mathrm{ED}$ visit was used to categorize patients by hyperkalemia severity (mild [ $>5.0-5.5 \mathrm{mEq} / \mathrm{L}]$, moderate [ $>5.5-6.0 \mathrm{mEq} / \mathrm{L}$ ], or severe $[>6.0 \mathrm{mEq} / \mathrm{L}])$. The ED admission date was required to be at least 6 months after the start of the data and the ED discharge date was required to be at least 90 days prior to the end of the data. Patients were also required to have at least one additional encounter after the discharge from the ED.

For patients with multiple eligible ED admissions with hyperkalemia, the index ED visit was randomly selected. The index date was defined as the admission date for the index ED visit with hyperkalemia. The baseline period was defined as the 6-month period prior to the index date. The period spanning from the index date to the date of ED discharge was defined as the ED visit period. The period up to 90 days after ED discharge was defined as the post-discharge period.

\section{Study Variables}

During the 6-month baseline period, patient characteristics, comorbidities (identified using the International Classification of DiseasesNinth/Tenth Revision [ICD-9, ICD-10] codes), previous hyperkalemia treatments (identified using RxNorm codes), and potassium laboratory values were described (Supplementary Table 1). Potassium levels, treatments, and monitoring were described during the ED visit period.

\section{Outcome Measures}

Death during the ED visit and the proportion of patients immediately admitted to inpatient care from the ED (i.e., admitted to inpatient care on 
the same day as their discharge from the ED) were reported. Among patients alive and not immediately admitted to inpatient care, hyperkalemia recurrence (defined as at least one potassium laboratory value $>5.0 \mathrm{mEq} / \mathrm{L}$ in any setting) was described within 30, 60, and 90 days of discharge from the ED. All-cause inpatient admissions and inpatient admissions with hyperkalemia (defined as an inpatient admission with at least one potassium laboratory value $>5.0 \mathrm{mEq} / \mathrm{L}$ ) were also described within 30, 60, and 90 days of ED discharge.

\section{Statistical Analysis}

Study variables and outcome measures were described and compared among patients with mild, moderate, and severe hyperkalemia. Counts and percentages were provided for categorical variables and means and standard deviations (SD) were provided for continuous variables. Chi-squared tests for categorical variables and analysis of variance tests for continuous variables were used to compare outcomes in patients with mild hyperkalemia to patients with moderate hyperkalemia and patients with mild hyperkalemia to patients with severe hyperkalemia; $p<0.05$ was considered statistically significant. All statistical analyses were conducted using SAS 9.4.

\section{RESULTS}

\section{Baseline Patient Characteristics by Hyperkalemia Severity}

A total of 6222 patients admitted to the ED with hyperkalemia were eligible for the analysis, among whom $4432(71.2 \%)$ patients had mild hyperkalemia, 1085 (17.4\%) patients had moderate hyperkalemia, and 705 (11.3\%) patients had severe hyperkalemia (Table 1 ). The mean ages of patients with mild, moderate, and severe hyperkalemia were similar (mild, 61.9; moderate, 62.1; severe, 62.0 years). About half of patients were female (mild, 51.7\%; moderate, $51.9 \%$; severe, $47.8 \%$ ). Overall, the majority of patients were White (49.0\%) or African American $(49.4 \%)$.

Comorbidities were common in patients with an ED visit with hyperkalemia, with an average Charlson comorbidity index (CCI) of 1.9 in mild patients, 2.2 in moderate patients, and 2.4 in severe patients (mild vs. moderate $p<0.001$, mild vs. severe $p<0.001$ ). Hypertension was the most common comorbidity, followed by CKD stage 3-5, type 2 diabetes, and heart failure. Rates of CKD stage 3-5, end-stage renal disease, acute kidney injury, and type 2 diabetes increased with severity of hyperkalemia. Heart failure, hypertension, and prior renin-angiotensin-aldosterone system inhibitor use were similar among patients in all hyperkalemia severities (Table 1).

\section{Comparison of Clinical Characteristics Across Hyperkalemia Severities During the Emergency Department Visit}

Most patients with mild hyperkalemia (85.0\%) and over half of patients with moderate hyperkalemia (59.6\%) had only one potassium laboratory assessment during their $\mathrm{ED}$ visit (Table 2). However, most patients with severe hyperkalemia $(75.5 \%)$ had at least two potassium laboratory assessments. Among patients with multiple potassium laboratory values, potassium levels returned to $\leq 5.0 \mathrm{mEq} / \mathrm{L}$ in over half of all patients (mild, 59.7\%; moderate, 65.5\%; severe, $57.1 \%$ ).

During the ED visit, patients with more severe hyperkalemia received more hyperkalemia treatments, which included potassium-binding treatments, temporizing agents, diuretics, or dialysis (mild, 13.4\%; moderate, 30.8\%; severe, $45.7 \%$; all $p<0.001$ ). Temporizing agents were the most common treatment and were more commonly used by patients with severe hyperkalemia (31.6\%). Sodium polystyrene sulfonate (SPS) treatment was reserved mostly for patients with severe hyperkalemia (severe $27.4 \%$ vs. mild $4.1 \%, p<0.001$ ). Diuretics, dialysis, and patiromer were not commonly used during the ED visit $(5.7 \%, 0.8 \%, 0.0 \%$ overall, respectively). Among patients with available ECG data, the proportion of patients receiving ECG 
Table 1 Baseline characteristics among patients with mild, moderate, and severe hyperkalemia during the 6 months prior to the ED visit

\begin{tabular}{|c|c|c|c|c|c|}
\hline & \multirow{2}{*}{$\begin{array}{l}\text { Mild } \\
\text { hyperkalemia } \\
>5.0-5.5 \mathrm{mEq} / \mathrm{L} \\
(N=4432)\end{array}$} & \multirow{2}{*}{$\begin{array}{l}\text { Moderate } \\
\text { hyperkalemia } \\
>5.5-6.0 \mathrm{mEq} / \mathrm{L} \\
(N=1085)\end{array}$} & \multirow{2}{*}{$\begin{array}{l}\text { Severe } \\
\text { hyperkalemia } \\
>6.0 \mathrm{mEq} / \mathrm{L} \\
(N=705)\end{array}$} & \multicolumn{2}{|l|}{$p$ values } \\
\hline & & & & $\begin{array}{l}\text { Mild vs. } \\
\text { moderate }\end{array}$ & $\begin{array}{l}\text { Mild vs. } \\
\text { severe }\end{array}$ \\
\hline \multicolumn{6}{|l|}{ Demographics } \\
\hline Age (years), mean (SD) & $61.9(18.5)$ & $62.1(18.0)$ & $62.0(17.7)$ & 0.76 & 0.97 \\
\hline Female, $n(\%)$ & $2292(51.7 \%)$ & $563(51.9 \%)$ & $337(47.8 \%)$ & 0.94 & 0.06 \\
\hline Race, $n(\%)$ & & & & $<0.05$ & $<0.05$ \\
\hline White & $2178(49.1 \%)$ & $486(44.8 \%)$ & $382(54.2 \%)$ & & \\
\hline Black or African American & $2185(49.3 \%)$ & $579(53.4 \%)$ & $307(43.5 \%)$ & & \\
\hline Asian, other, or missing & $69(1.5 \%)$ & $20(1.8 \%)$ & $16(2.3 \%)$ & & \\
\hline \multicolumn{6}{|l|}{ Comorbidities } \\
\hline CKD (stage 3-5), $n$ (\%) & $1768(39.9 \%)$ & $515(47.5 \%)$ & $353(50.1 \%)$ & $<0.001$ & $<0.001$ \\
\hline $\begin{array}{l}\text { CKD stage among patients } \\
\text { with CKD, } n(\%)\end{array}$ & & & & $<0.05$ & $<0.05$ \\
\hline Stage 3 & $942(53.3 \%)$ & $240(46.6 \%)$ & $171(48.4 \%)$ & & \\
\hline Stage 4 & $305(17.3 \%)$ & $92(17.9 \%)$ & $54(15.3 \%)$ & & \\
\hline Stage 5 or ESRD & $521(29.5 \%)$ & $183(35.5 \%)$ & $128(36.3 \%)$ & & \\
\hline $\mathrm{ESRD}, n(\%)$ & $228(5.1 \%)$ & $91(8.4 \%)$ & $64(9.1 \%)$ & $<0.001$ & $<0.001$ \\
\hline Acute kidney injury, $n$ (\%) & $633(14.3 \%)$ & $179(16.5 \%)$ & $154(21.8 \%)$ & 0.07 & $<0.001$ \\
\hline Type 2 diabetes, $n$ (\%) & $1618(36.5 \%)$ & $431(39.7 \%)$ & $288(40.9 \%)$ & 0.05 & $<0.05$ \\
\hline Heart failure, $n(\%)$ & $959(21.6 \%)$ & $264(24.3 \%)$ & $154(21.8 \%)$ & 0.06 & 0.94 \\
\hline Hypertension, $n(\%)$ & $2731(61.6 \%)$ & $708(65.3 \%)$ & $452(64.1 \%)$ & $<0.05$ & 0.22 \\
\hline RAASi use, $n(\%)$ & $1378(31.1 \%)$ & $344(31.7 \%)$ & $230(32.6 \%)$ & 0.72 & 0.44 \\
\hline $\begin{array}{l}\text { Charlson Comorbidity Index } \\
\text { (CCI), mean (SD) }\end{array}$ & $1.9(2.3)$ & $2.2(2.4)$ & $2.4(2.5)$ & $<0.001$ & $<0.001$ \\
\hline \multicolumn{6}{|l|}{ Previous treatments used, $n$ (\%) } \\
\hline SPS or patiromer & $238(5.4 \%)$ & $102(9.4 \%)$ & $89(12.6 \%)$ & $<0.001$ & $<0.001$ \\
\hline Diuretics & $1267(28.6 \%)$ & $319(29.4 \%)$ & $275(39.0 \%)$ & 0.62 & $<0.001$ \\
\hline Dialysis & $241(5.4 \%)$ & $96(8.8 \%)$ & $71(10.1 \%)$ & $<0.001$ & $<0.001$ \\
\hline \multicolumn{6}{|l|}{ Potassium labs during baseline } \\
\hline Number of potassium labs, mean (SD) & $5.9(12.7)$ & $7.6(15.7)$ & $11.0(22.0)$ & $<0.001$ & $<0.001$ \\
\hline
\end{tabular}


Table 1 continued

\begin{tabular}{|c|c|c|c|c|c|}
\hline & \multirow{2}{*}{$\begin{array}{l}\text { Mild } \\
\text { hyperkalemia } \\
>5.0-5.5 \mathrm{mEq} / \mathrm{L} \\
(N=4432)\end{array}$} & \multirow{2}{*}{$\begin{array}{l}\text { Moderate } \\
\text { hyperkalemia } \\
>5.5-6.0 \mathrm{mEq} / \mathrm{L} \\
(N=1085)\end{array}$} & \multirow{2}{*}{$\begin{array}{l}\text { Severe } \\
\text { hyperkalemia } \\
>6.0 \mathrm{mEq} / \mathrm{L} \\
(N=705)\end{array}$} & \multicolumn{2}{|l|}{$p$ values } \\
\hline & & & & $\begin{array}{l}\text { Mild vs. } \\
\text { moderate }\end{array}$ & $\begin{array}{l}\text { Mild vs. } \\
\text { severe }\end{array}$ \\
\hline $\begin{array}{l}\text { Proportion with at least } 1 \\
\text { potassium lab, } n(\%)\end{array}$ & $2948(66.5 \%)$ & $757(69.8 \%)$ & $487(69.1 \%)$ & $<0.05$ & 0.18 \\
\hline \multicolumn{6}{|l|}{ Among patients with at least 1 potassium lab } \\
\hline Any potassium labs $>5 \mathrm{mEq} / \mathrm{L}, n(\%)$ & $543(18.4 \%)$ & $211(27.9 \%)$ & $189(38.8 \%)$ & $<0.001$ & $<0.001$ \\
\hline $\begin{array}{l}\text { Number of potassium labs }>5 \mathrm{mEq} / \mathrm{L} \text {, } \\
\text { mean }(\mathrm{SD})\end{array}$ & $1.1(3.0)$ & $1.8(3.6)$ & $3.0(5.7)$ & $<0.001$ & $<0.001$ \\
\hline
\end{tabular}

$p$ values for categorical variables were calculated using chi-squared tests; $p$ values for continuous variables were calculated using analysis of variance (ANOVA) tests

$C K D$ chronic kidney disease, ED emergency department, ESRD end-stage renal disease, $N$ number, RAASi renin-angiotensin-aldosterone system inhibitor, $S D$ standard deviation, SPS sodium polystyrene sulfonate

monitoring increased with hyperkalemia severity (mild, 56.5\%; moderate, 69.6\%; severe, $81.0 \%$; mild vs. moderate $p<0.001$, mild vs. severe $p<0.001)$. At ED discharge, only a small proportion of patients received treatment with a potassium-binding treatment (SPS or patiromer) (mild, 0.03\%; moderate, $1.9 \%$; severe, $5.2 \%$; mild vs. moderate $p<0.001$, mild vs. severe $p<0.001$ ).

\section{Emergency Department and Post- Discharge Outcomes Across Hyperkalemia Severities}

A total of $10.6 \%$ of patients with severe hyperkalemia died during their ED visit, compared with $3.7 \%$ of patients with moderate hyperkalemia and $1.1 \%$ with mild hyperkalemia (mild vs. moderate $p<0.001$; mild vs. severe $p<0.001)$. The proportion of patients directly admitted to inpatient care increased as hyperkalemia severity increased (mild, 5.8\%; moderate, $8.7 \%$; severe, $12.7 \%$; mild vs. moderate $p<0.001$, mild vs. severe $p<0.001$ ) (Table 2).

Among patients not directly admitted to inpatient care, the proportion of patients with hyperkalemia recurrence within 30 days post- discharge increased with hyperkalemia severity (mild, 12.9\%; moderate, 19.0\%; severe, 32.5\%; mild vs. moderate hyperkalemia $p<0.001$, mild vs. severe hyperkalemia $p<0.001)$. Similar patterns were observed within 60 and 90 days postdischarge from the ED (Fig. 1). Across all severity cohorts, all-cause inpatient admissions were similar 30 days post-discharge (mild, 14.9\%; moderate, 15.8\%; severe, 14.0\%). A similar trend was observed for all-cause inpatient admissions 60 and 90 days post-discharge (Fig. 1). The proportion of patients with inpatient admissions with hyperkalemia 30 days post-discharge increased with hyperkalemia severity (mild, 6.5\%; moderate, 7.9\%; severe, $9.3 \%$; mild vs. moderate hyperkalemia $p=0.16$, mild vs. severe $p<0.05$ ) with similar trends observed within 60 and 90 days post-discharge (Fig. 1).

\section{DISCUSSION}

This study comprehensively characterized the patient demographics and clinical characteristics, hyperkalemia monitoring and management practices, and post-discharge outcomes 
Table 2 Comparison of clinical characteristics among patients with mild, moderate, and severe hyperkalemia during the ED visit

\begin{tabular}{|c|c|c|c|c|c|}
\hline & \multirow{2}{*}{$\begin{array}{l}\text { Mild } \\
\text { hyperkalemia } \\
>5.0-5.5 \mathrm{mEq} / \mathrm{L} \\
(N=4432)\end{array}$} & \multirow{2}{*}{$\begin{array}{l}\text { Moderate } \\
\text { hyperkalemia } \\
>5.5-6.0 \mathrm{mEq} / \mathrm{L} \\
(N=1085)\end{array}$} & \multirow{2}{*}{$\begin{array}{l}\text { Severe } \\
\text { hyperkalemia } \\
>6.0 \mathrm{mEq} / \mathrm{L} \\
(N=705)\end{array}$} & \multicolumn{2}{|l|}{$p$ values } \\
\hline & & & & $\begin{array}{l}\text { Mild vs. } \\
\text { moderate }\end{array}$ & $\begin{array}{l}\text { Mild vs } \\
\text { severe }\end{array}$ \\
\hline \multicolumn{6}{|l|}{ Death during ED visit, $n(\%)$} \\
\hline Patients who died & $50(1.1 \%)$ & $40(3.7 \%)$ & $75(10.6 \%)$ & $<0.001$ & $<0.001$ \\
\hline \multicolumn{6}{|l|}{ Potassium lab values during ED visit } \\
\hline $\begin{array}{l}\text { Number of potassium lab values, } \\
\text { mean }(\mathrm{SD})\end{array}$ & $1.2(0.5)$ & $1.5(0.9)$ & $2.2(1.4)$ & $<0.001$ & $<0.001$ \\
\hline $\begin{array}{l}\text { Patients with } \geq 2 \text { potassium lab } \\
\text { values, } n(\%)\end{array}$ & $663(15.0 \%)$ & $438(40.4 \%)$ & $532(75.5 \%)$ & $<0.001$ & $<0.001$ \\
\hline \multicolumn{6}{|l|}{ Among patients with $\geq 2$ lab values } \\
\hline $\begin{array}{l}\text { First potassium lab value, mean } \\
\text { (SD) }\end{array}$ & $5.3(0.1)$ & $5.8(0.1)$ & $6.7(0.8)$ & $<0.001$ & $<0.001$ \\
\hline $\begin{array}{l}\text { Last potassium lab value, mean } \\
\text { (SD) }\end{array}$ & $4.8(0.6)$ & $4.8(0.7)$ & $4.9(1.0)$ & 0.96 & $<0.001$ \\
\hline $\begin{array}{l}\text { Potassium lab value returned } \\
\text { to } \leq 5.0 \mathrm{mEq} / \mathrm{L} \text { by the end } \\
\text { of the visit, } n(\%)\end{array}$ & $396(59.7 \%)$ & $287(65.5 \%)$ & $304(57.1 \%)$ & 0.06 & 0.4 \\
\hline \multicolumn{6}{|l|}{ Monitoring during ED visit, $n(\%)$} \\
\hline Electrocardiogram $^{\mathrm{a}}$ & $2151(56.5 \%)$ & $646(69.6 \%)$ & $532(81.0 \%)$ & $<0.001$ & $<0.001$ \\
\hline \multicolumn{6}{|l|}{ Treatment during ED visit, $n$ (\%) } \\
\hline Any treatment & $592(13.4 \%)$ & $334(30.8 \%)$ & $322(45.7 \%)$ & $<0.001$ & $<0.001$ \\
\hline Any temporizing agent & $248(5.6 \%)$ & $168(15.5 \%)$ & $223(31.6 \%)$ & $<0.001$ & $<0.001$ \\
\hline Albuterol & $185(4.2 \%)$ & $84(7.7 \%)$ & $103(14.6 \%)$ & $<0.001$ & $<0.001$ \\
\hline Calcium & $53(1.2 \%)$ & $74(6.8 \%)$ & $134(19.0 \%)$ & $<0.001$ & $<0.001$ \\
\hline Insulin with glucose IV & $31(0.7 \%)$ & $76(7.0 \%)$ & $123(17.4 \%)$ & $<0.001$ & $<0.001$ \\
\hline Sodium bicarbonate IV & $44(1.0 \%)$ & $49(4.5 \%)$ & $97(13.8 \%)$ & $<0.001$ & $<0.001$ \\
\hline SPS & $183(4.1 \%)$ & $186(17.1 \%)$ & $193(27.4 \%)$ & $<0.001$ & $<0.001$ \\
\hline Patiromer & $0(0.0 \%)$ & $0(0.0 \%)$ & $0(0.0 \%)$ & - & - \\
\hline Diuretics & $225(5.1 \%)$ & $76(7.0 \%)$ & $53(7.5 \%)$ & $<0.05$ & $<0.05$ \\
\hline Dialysis & $19(0.4 \%)$ & $9(0.8 \%)$ & $21(3.0 \%)$ & 0.15 & $<0.001$ \\
\hline \multicolumn{6}{|c|}{ Potassium binding treatments at discharge, $n(\%)$} \\
\hline SPS at discharge & $15(0.3 \%)$ & $21(1.9 \%)$ & $37(5.2 \%)$ & $<0.001$ & $<0.001$ \\
\hline
\end{tabular}


Table 2 continued

\begin{tabular}{|c|c|c|c|c|c|}
\hline & \multirow{2}{*}{$\begin{array}{l}\text { Mild } \\
\text { hyperkalemia } \\
>5.0-5.5 \mathrm{mEq} / \mathrm{L} \\
(N=4432)\end{array}$} & \multirow{2}{*}{$\begin{array}{l}\text { Moderate } \\
\text { hyperkalemia } \\
>5.5-6.0 \mathrm{mEq} / \mathrm{L} \\
(N=1085)\end{array}$} & \multirow{2}{*}{$\begin{array}{l}\text { Severe } \\
\text { hyperkalemia } \\
>6.0 \mathrm{mEq} / \mathrm{L} \\
(N=705)\end{array}$} & \multicolumn{2}{|l|}{$p$ values } \\
\hline & & & & $\begin{array}{l}\text { Mild vs. } \\
\text { moderate }\end{array}$ & $\begin{array}{l}\text { Mild vs. } \\
\text { severe }\end{array}$ \\
\hline Patiromer at discharge & $0(0.0 \%)$ & $0(0.0 \%)$ & $0(0.0 \%)$ & - & - \\
\hline \multicolumn{6}{|l|}{ Direct admission to inpatient care, $n(\%)$} \\
\hline Admitted to inpatient care from ED & $252(5.8 \%)$ & $91(8.7 \%)$ & $80(12.7 \%)$ & $<0.001$ & $<0.001$ \\
\hline
\end{tabular}

$E D$ emergency department, $I V$ intravenous, $N$ number, $S D$ standard deviation, SPS sodium polystyrene sulfonate

${ }^{a}$ Electrocardiogram use was calculated among patients that had electrocardiogram data available (mild, $n=3808$; moderate, $n=928$; severe, $n=657)$; $p$ values for categorical variables were calculated using chi-squared tests; $p$ values for continuous variables were calculated using analysis of variance (ANOVA) tests

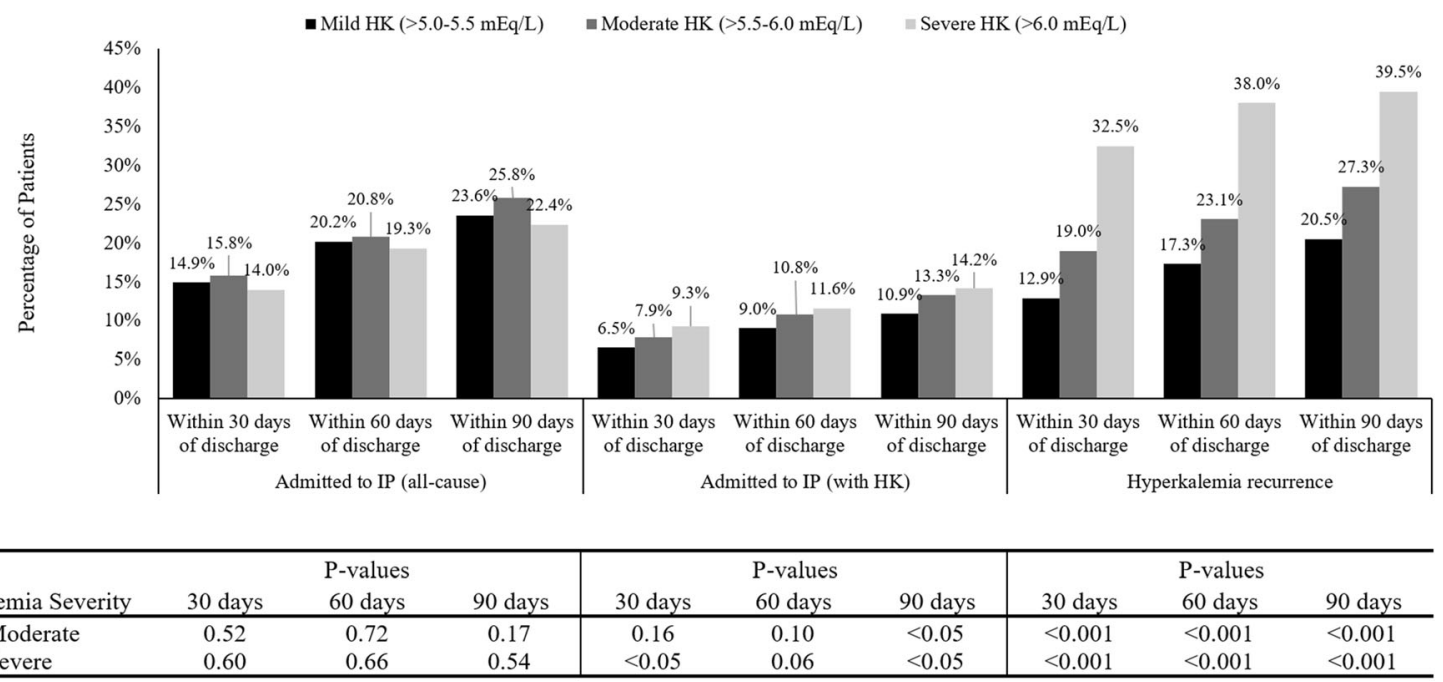

Fig. 1 Post-discharge events 30,60, and 90 days after ED visit by hyperkalemia severity among patients discharged from the ED ${ }^{a}$. ${ }^{a}$ Calculated among patients alive and not immediately admitted to discharge after the ED visit (mild, $n=4130$; moderate, $n=954$; severe, $n=550)$; $p$ values for categorical variables were calculated using chi-squared

among patients with hyperkalemia in the ED. This study found that in the ED setting, patients with moderate and severe hyperkalemia had elevated rates of death and immediate inpatient admission, using descriptive analyses. After ED discharge, these patients also had higher rates of inpatient admission and hyperkalemia recurrence. Given the arrival of the novel potassium binders, the management of hyperkalemia has tests; $p$ values for continuous variables were calculated using ANOVA tests. ED emergency department, $H K$ hyperkalemia, IP inpatient

entered a new era and this study provides a contemporary description of the ED management of patients with hyperkalemia by severity.

As a result of the potentially fatal consequences of severe hyperkalemia, patients with hyperkalemia are often managed in the ED $[11,18]$. The absence of robust and evidencebased treatment guidelines for hyperkalemia management in the ED has posed a challenge 
for treating hyperkalemia in the ED setting [14-16]. Prior treatment algorithms that have been published to aid in the clinical management of hyperkalemia in the ED were largely based on anecdotal experience and findings from a small number of patients $[8,14]$. However, a recent Kidney Disease: Improving Global Outcomes Controversies Conference was held where a multidisciplinary group composed of emergency medicine specialists, nephrologists, renal physiologists, endocrinologists, cardiologists, and dieticians convened to identify best practices in the clinical management of hyperkalemia [9]. In addition to consensus on an evidence-based treatment algorithm, this conference highlighted the need for frequent evaluations to assess treatment success [9].

ECG monitoring and repeated assessments of serum potassium levels is a common practice to monitor hyperkalemia, particularly in acute hyperkalemic episodes and among patients with comorbidities [4, 13, 19]. In this study, most patients with severe hyperkalemia were monitored via ECG and had repeated laboratory assessments of serum potassium levels, which aligns with previous studies $[16,18,20]$. Temporizing agents (e.g., albuterol, calcium, etc.) were administered to $16-32 \%$ of patients with moderate and severe hyperkalemia during the ED visit. Although temporizing agents are helpful in stabilizing cardiac function, they do not eliminate excess potassium from the body $[3,14,19]$. After treatment with a temporizing agent, SPS was the most commonly used treatment. However, SPS provides limited value in the short-term management of hyperkalemia and it is occasionally associated with severe adverse events (e.g., colonic necrosis and intense diarrhea) $[14,15]$. The study results pointed to a clear unmet need in treatment of patients with hyperkalemia in the ED setting.

The proportion of patients who died during the ED visit increased as the severity of hyperkalemia increased (mild, 1.1\%; moderate, 3.7\%; severe, $10.6 \%$; all $p<0.001$ ). Many previous studies $[2,16,21]$ also reported an increase in mortality with increased hyperkalemia severity. For example, a 2017 retrospective study found that compared to patients with normal potassium levels $(3.5-5.0 \mathrm{mEq} / \mathrm{L})$, patients with "severely elevated" potassium levels (> $6.0 \mathrm{mEq} / \mathrm{L}$ ) had five times higher odds of dying (95\% confidence interval 2.9-8.6) [16]. However, a recent study found that reducing potassium levels to $<5.5 \mathrm{mEq} / \mathrm{L}$ in the $\mathrm{ED}$ was associated with a $50 \%$ mortality reduction after adjusting for age, creatinine, comorbidities, and initial potassium level, which highlighted the importance of appropriate management of hyperkalemia in the ED setting [17].

At the end of the ED visit, $34-43 \%$ of the patients still had elevated potassium levels (i.e., $>5.0 \mathrm{mEq} / \mathrm{L}$ ). However, at discharge, only a small proportion of patients (less than $6 \%$ in the severe group) received treatment with a potassium-binding treatment (SPS or patiromer). The gap between the elevated potassium level at the end of the ED visit and the low prescription rate of hyperkalemia treatment at discharge may indicate an unmet need for post-ED management of hyperkalemia. The post-discharge burden of hyperkalemia recurrence and hyperkalemia-related inpatient admission further demonstrate such an unmet need.

Using a large EMR database, this study characterized ED management and post-discharge outcomes across hyperkalemia severities; future studies that examine predictors of postED outcomes including hyperkalemia recurrence and inpatient admission would be valuable. Furthermore, as the awareness of the unmet need of patients with hyperkalemia increases, in both the ED and post-discharge settings [22], there is an impetus to understand how clinicians can more effectively treat patients within the ED and after discharge from the ED to ultimately lower rates of hyperkalemia recurrence and readmission. With the development of novel potassium binders [23], future studies are needed to identify which patient groups (e.g., those with moderate to severe hyperkalemia or with recurring hyperkalemia) would benefit the most from emerging therapies.

\section{Limitations}

The findings in this study offer a valuable benchmark that can help healthcare 
stakeholders assess progress in the clinical management of hyperkalemia. This study, conducted in a large EMR database, provides a rich data source including laboratory data and longitudinal information on patients with care across multiple settings, which enabled a characterization of events that occurred both during and after an ED visit. One of the limitations of the study is that although we assessed a large number of variables at baseline and during the ED visit, not all clinical characteristics were assessed such as serum bicarbonate level. Despite that recent meta-analysis [24] has shown that risk of hyperkalemia and mortality among patients with ESRD can be partially dependent on the duration of between-dialysis period, we found the proportion of stage 5 or patients with ESRD increased as hyperkalemia severity increases. However, we did not have the data on duration of between-dialysis period to provide additional insights into this patient population with ESRD.

Although the large number of patients in this study likely comprise a representative sample of patients with hyperkalemia in Louisiana, the generalizability of this study may be limited if the management of hyperkalemia in Louisiana differs from other areas in the US. Additionally, because out-of-network healthcare encounters were not captured in the EMR database, the baseline and post-discharge results in this study may be slightly underestimated.

\section{CONCLUSIONS}

Patients with moderate and severe hyperkalemia experienced elevated risk of death and direct inpatient admission after an ED visit, as well as hyperkalemia recurrence and hyperkalemia-related inpatient readmissions following discharge from the $\mathrm{ED}$, from a descriptive analysis. Additional studies are warranted to assess strategies to reduce recurrence and inpatient admission in this patient population.

\section{ACKNOWLEDGEMENTS}

Funding. Sponsorship for this study and the journal's Rapid Service Fees were funded by AstraZeneca.

Medical Writing, Editorial, and Other Assistance. Editorial assistance in the preparation of this article was provided by Dr. Gloria DeWalt of Analysis Group, Inc. Support for this assistance was funded by AstraZeneca.

Authorship. All named authors meet the International Committee of Medical Journal Editors (ICMJE) criteria for authorship for this article, take responsibility for the integrity of the work as a whole, and have given their approval for this version to be published.

Author Contributions. Jill Davis, Rubeen Israni, Keith A. Betts, Fan Mu, Erin E. Cook, Deborah Anzalone, Harold Szerlip, Lei Yin, Gabriel I. Uwaifo, and Eric Q. Wu contributed to the study's design, data acquisition, analysis, and interpretation. Keith A. Betts, Fan Mu, Erin E. Cook, and Eric Q. Wu had a significant role in conducting formal analyses. All authors contributed to writing and editing of the manuscript.

Disclosures. Jill Davis, Rubeen Israni, and Deborah Anzalone were employees of AstraZeneca at the time the study was conducted. Jill Davis is currently affiliated with ENDO Pharmaceuticals, Rubeen Israni is currently affiliated with GlaxoSmithKline, and Deborah Anzalone has since retired and so has no current affiliation. Keith A. Betts, Fan Mu, Erin E. Cook, Lei Yin, and Eric Q. Wu are employees of Analysis Group, Inc., which received payment from AstraZeneca for the conduct of this analysis. Harold Szerlip is on the advisory board and speaker's bureau for AstraZeneca. Gabriel I. Uwaifo has nothing to disclose.

Compliance with Ethics Guidelines. The New England Independent Review Board provided written approval for this study on June 25, 2018 (NEIRB\# 1-5667-1). Written and 
verbal consent from participants are not applicable to this study.

Data Availability. The datasets analyzed in this study are not publicly available. The research reported in this article was conducted in partnership with Research Action for Health Network (REACHnet), funded by the Patient Centered Outcomes Research Institute ${ }^{\circledR}$ (PCORI Award RI-CRN-2020-008). REACHnet is a partner network in PCORnet ${ }^{\circledR}$, the National PatientCentered Clinical Research Network, which was developed with funding from PCORI ${ }^{\circledR}$. The content of this article is solely the responsibility of the author(s) and does not necessarily represent the views of other organizations participating in, collaborating with, or funding REACHnet or PCORnet ${ }^{\circledR}$, or of PCORI ${ }^{\circledR}$. The authors acknowledge the participation of REACHnet partner health systems: Tulane Medical Center and Ochsner Medical Center in this project.

Open Access. This article is licensed under a Creative Commons Attribution-NonCommercial 4.0 International License, which permits any non-commercial use, sharing, adaptation, distribution and reproduction in any medium or format, as long as you give appropriate credit to the original author(s) and the source, provide a link to the Creative Commons licence, and indicate if changes were made. The images or other third party material in this article are included in the article's Creative Commons licence, unless indicated otherwise in a credit line to the material. If material is not included in the article's Creative Commons licence and your intended use is not permitted by statutory regulation or exceeds the permitted use, you will need to obtain permission directly from the copyright holder. To view a copy of this licence, visit http://creativecommons.org/licenses/by$\mathrm{nc} / 4.0 /$.

\section{REFERENCES}

1. Lehnhardt A, Kemper MJ. Pathogenesis, diagnosis and management of hyperkalemia. Pediatr Nephrol. 2011;26(3):377-84.

2. Luo J, Brunelli SM, Jensen DE, Yang A. Association between serum potassium and outcomes in patients with reduced kidney function. Clin J Am Soc Nephrol. 2016;11(1):90-100.

3. Mushiyakh Y, Dangaria H, Qavi S, et al. Treatment and pathogenesis of acute hyperkalemia. J Community Hosp Intern Med Perspect. 2012. https:// doi.org/10.3402/jchimp.v1i4.7372.

4. Hollander-Rodrigues JC, Calvert JF. Hyperkalemia. Am Fam Physician. 2006;73(2):283-90.

5. Betts KA, Woolley JM, Mu F, et al. The prevalence of hyperkalemia in the United States. Curr Med Res Opin. 2018;34(6):971-8.

6. Dorans KS, Mills KT, Liu Y, He J. Trends in prevalence and control of hypertension according to the 2017 American College of Cardiology/American Heart Association (ACC/AHA) Guideline. J Am Heart Assoc. 2018. https://doi.org/10.1161/JAHA. 118.008888 .

7. Betts KA, Woolley JM, Mu F, et al. The cost of hyperkalemia in the United States. Kidney Int Rep. 2018;3(2):385-93.

8. Rossignol P, Legrand M, Kosiborod M, et al. Emergency management of severe hyperkalemia: guideline for best practice and opportunities for the future. Pharmacol Res. 2016;113:585-91.

9. Lindner G, Burdmann EA, Clase CM, et al. Acute hyperkalemia in the emergency department: a summary from a Kidney Disease: Improving Global Outcomes conference. Eur J Emerg Med. 2020;27(5):329-37.

10. Ahee $\mathrm{P}$, Crowe AV. The management of hyperkalaemia in the emergency department. J Accid Emerg Med. 2000;17(3):188-91.

11. Kovesdy CP. Management of hyperkalemia: an update for the internist. Am J Med. 2015;128(12): 1281-7.

12. Acker CG, Johnson JP, Palevsky PM, Greenberg A. Hyperkalemia in hospitalized patients: causes, adequacy of treatment, and results of an attempt to improve physician compliance with published therapy guidelines. Arch Intern Med. 1998;158(8): 917-24. 
13. Lane D. 2008. Hyperkalemia, Society for Academic Emergency Medicine Philadelphia, Pennsylvania: Temple University School of Medicine. https:// www.saem.org/cdem/education/online-education/ m4-curriculum/group-m4-endocrine-electrolytes/ hyperkalemia. Accessed 6 June 2019.

14. Rafique Z, Chouihed T, Mebazaa A, Frank PW. Current treatment and unmet needs of hyperkalaemia in the emergency department. Eur Heart J Suppl. 2019;21(Suppl A):A12-9.

15. Peacock WF, Rafique Z, Clark CL, et al. Real world evidence for treatment of hyperkalemia in the emergency department (REVEAL-ED): a multicenter, prospective, observational study. J Emerg Med. 2018;55(6):741-50.

16. Singer AJ, Thode HC Jr, Peacock WF. A retrospective study of emergency department potassium disturbances: severity, treatment, and outcomes. Clin Exp Emerg Med. 2017;4(2):73-9.

17. Singer AJ, Thode HC, Peacock WF. Rapid correction of hyperkalemia is associated with reduced mortality in ED patients. Am J Emerg Med. 2019;38(11): 2361-64.

18. Freeman K, Feldman JA, Mitchell P, et al. Effects of presentation and electrocardiogram on time to treatment of hyperkalemia. Acad Emerg Med. 2008;15(3):239-49.
19. National Kidney Foundation. 2016. Best practices in managing hyperkalemia in chronic kidney disease. https://www.kidney.org/sites/default/files/0210-7259\%20Hyperkalemia\%20Tool.pdf. Accessed 23 Aug 2019.

20. Pfortmüller CA, Leichtle AB, Fiedler GM, Exadaktylos AK, Lindner G. Hyperkalemia in the emergency department: etiology, symptoms and outcome of a life threatening electrolyte disorder. Eur J Intern Med. 2013;24(5):e59-60.

21. Goyal A, Spertus JA, Gosch K, et al. Serum potassium levels and mortality in acute myocardial infarction. JAMA. 2012;307(2):157-64.

22. Aggarwal S, Topaloglu H, Kumar S. Trends in emergency room visits due to hyperkalemia in the United States. Value Health. 2015;18:A386.

23. Rossignol P. A new area for the management of hyperkalaemia with potassium binders: clinical use in nephrology. Eur Heart J Suppl. 2019;21(Suppl A): A48-54.

24. Bem D, Sugrue D, Wilding B, et al. The effect of hyperkalemia and long inter-dialytic interval on morbidity and mortality in patients receiving hemodialysis: a systematic review. Ren Fail. 2021;43(1):241-54. 